\title{
Management of FLT-3 mutated AML
}

\author{
Dr. Nour El Hoda Hussein \\ Lecturer of Internal Medicine, \\ Department of Hematology and Bone Marrow transplantation, \\ Ain Shams University
}

\section{Introduction}

Acute myeloid leukemia (AML) accounts for approximately $30 \%$ of leukemia's but $>40 \%$ of leukemia-related deaths. ${ }^{1} \mathrm{AML}$ is highly diverse and cytogenetic analysis of metaphase cells reveals that approximately $40-50 \%$ of patients with de novo AML have a normal karyotype. The clinical outcomes for these AML patients with a normal karyotype are heterogeneous. Mutations in key genes involved in AML pathogenesis lead to large inter patient variability in prognosis and outcomes. Some of these mutations like the internal tandem duplication (ITD) in the FMS-like tyrosine kinase receptor-3 (FLT3) gene, partial tandem duplication in the mixed lineage leukemia gene, Wilm's tumor gene mutations and overexpression of the BAALC gene are associated with poor prognosis in AML. ${ }^{2}$ On the other hand, CCAAT/enhancerbinding protein $\alpha$ (CEBPA) and nucleophosmin 1 (NPM1) mutations (in FLT3-ITD-negative patients) have been associated with improved prognosis. ${ }^{3}$

\section{FLT3 Receptor: Biology}

The fms-like tyrosine kinase (FLT3) is a type III receptor tyrosine kinase in the PDGF family of growth factor receptors. It was originally cloned from $\mathrm{CD}_{4} 4^{+}$hematopoietic stem cells more than a decade ago by two independent group-. FLT3 has an extracellular ligand-binding region with five immunoglobulin-like domains and a single transmembrane domain (Figure 1).

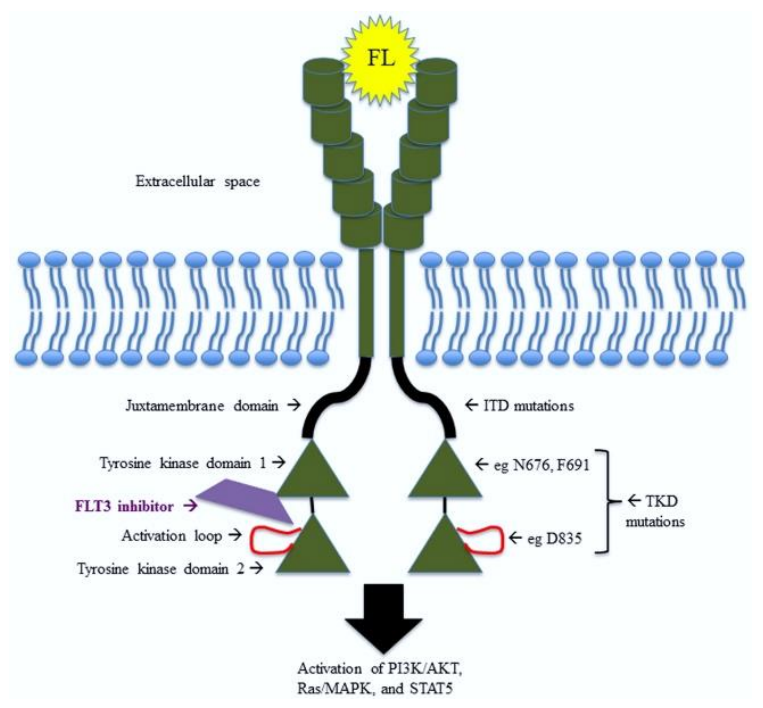


The cytoplasmic portion is comprised of a juxtamembrane domain followed by the catalytic domain, which is interrupted by a kinase insert domain. FLT3 ligand (FL) is a type I transmembrane protein expressed on the surface of stromal and hematopoietic cells in the bone marrow and in virtually all cell types examined. FL binding induces FLT3 conformational changes, dimerization and activation by cross-phosphorylation of intracytoplasmic tyrosine residues. This autophosphorylation results in the transduction of growth-promoting and apoptosis-inhibitory signals through pathways linked to key cytoplasmic molecules including RAS-GAP, PLC- $\beta$, STAT5 and ERK1/2.

In contrast to its ligand (FL, which seems to be ubiquitously expressed), FLT3 has a narrow tissue-restricted pattern of expression, being primarily localized to hematopoietic and neural tissues, and this confines its function to these tissue compartments. In the bone marrow, FLT3 is expressed predominantly by $\mathrm{CD}_{3} 4^{+}$hematopoietic cells and is considered a key receptor in hematopoietic development. Its activation synergizes with other signaling pathways to promote hematopoietic precursor expansion. Targeted disruption of either FLT3 or its ligand results in nonlethal quantitative deficiency in primitive hematopoietic precursors as well as lymphoid and dendritic cells. ${ }^{4}$

\section{FLT3 Receptor in AML: Molecular Genetics and Clinical Aspects}

The exact frequency of FLT3 varies with age with mutations being present in approximately 20 percent of patients with cytogenetically normal AML. There are two major types of FLT3 mutations. The most common is an ITD on exon 14 of the FLT3 gene, which varies from 3 to $>400$ base pairs. It is typically in frame and occurs in the juxta-membrane region of the receptor. This ITD disrupts the auto inhibitory function of the juxta-membrane domain and results in ligand-independent activation of the FLT3 receptor. This leads to a proliferative signal via activation of its downstream effectors, and produces growth factor-independent proliferation in leukemia cell lines and a fatal myeloproliferative syndrome in murine models. Thus, the ITD leads to gain of function mainly by inducing hyperresponsivity of the FLT3 receptor to FL rather than through auto-activation of the receptor. ${ }^{5}$

FLT3 kinase domain mutations (FLT3 TKDmut), which are found in about 7\% of newly diagnosed AML, seem to have limited impact on clinical outcomes; therefore attention has been primarily focused on developing improved therapies for FLT3-ITD AML. ${ }^{2}$

TKD FLT3 mutations are found in only $7 \%$ of AML, $3 \%$ of myelodysplastic syndrome (MDS) and $3 \%$ of acute lymphoblastic leukemia. FLT3-ITD is readily detected by PCR amplification of genomic DNA using primers that span the internal tandem repeat region.

Confirming the presence of FLT3 mutation in AML is now an integral part of the prognostic evaluation of newly diagnosed disease, particularly in the presence of normal cytogenetics. In addition to the poor outcome, a FLT3-ITD is associated with clinical features including hyperleukocytosis, rapid proliferation rate and hypercellular bone marrow packed with blasts.

While the rate of achieving a complete remission does not seem to be altered by a FLT3-ITD, its presence impacts many relevant end points, including the length of remission, event-free survival 
and overall survival. Additionally, the prognostically adverse phenotype imparted by FLT3-ITD negates the good prognostic value of a concurrent nucleophosmin mutation in AML with normal karyotype.

FLT3-ITD-expressing murine and human myeloid cell lines are resistant to cytosine arabinoside. Additional, in vitro evidence also suggests that FLT3-ITD may contribute to drug resistance. This has led to intensive research to identify therapeutic inhibitors of FLT3. Several of these agents are currently in clinical trials and have shown promising anti-leukemic activity. ${ }^{5}$

\section{FLT3 inhibitors in clinical trials lestaurtinib (CEP701)}

Several small molecule inhibitors of tyrosine kinases were studied in early phase clinical studies. One of the most studied early agents in development is lestaurtinib (CEP701) with a phase $1 / 2$ trial of lestaurtinib in relapsed or refractory AML patients with FLT3 mutations.

Correlative assays in this and a subsequent phase 2 study demonstrated that clinical response was more likely in patients who had in vitro leukemic blast sensitivity to CEP-701, and if, in vivo, CEP701 in plasma level was sufficient to significantly inhibit FLT3 autophosphorylation in a sustained fashion. Partial response was achieved in 8 of 27 patients ( 3 of 5 FLT3 ITD. 5 of 22 WT) All 8 responders had drug plasma levels sufficient to inhibit FLT3 phosphorylation to below $15 \%$ of baseline activity. ${ }^{4}$

\section{Midostaurin (PKC412)}

Midostaurin, an indolocarbazole derivative like lestaurtinib was evaluated in a phase II trial for relapsed or refractory FLT3 mutated AML patients. ${ }^{6}$ At a dose of $75 \mathrm{mg}$ three times daily, 14/20 patients displayed at least hematologic improvement, with $1 \mathrm{CR}$. Midostaurin is tightly bound to Alpha-1 Acid Glycoprotein (AAG) and responses correlated very well with the degree of FLT3 inhibition determined by the pharmacodynamic assessment of FLT3 inhibitory activity in the patient's plasma (PIA). ${ }^{7}$

\section{Sorafenib}

Sorafenib (bi-aryl urea) is multi-kinase inhibitor, approved for use in renal cell carcinoma, has been evaluated in early phase clinical trials. As a single agent, sorafenib has been studied on an intermittent schedule in refractory AML with or without a FLT3 mutation. A clinical response was observed in 9/16 patients (56\%) including all 6 patients with FLT3-ITD as a solitary. ${ }^{8}$ In a separate Phase I dose escalation trial of sorafenib in relapsed/refractory acute leukemias, PIA of kinase targets ERK and FLT3-ITD demonstrated excellent target inhibition, with FLT3-ITD silencing occurring below the MTD. ${ }^{9}$

\section{Quizartinib (AC220)}

A novel bis-aryl urea, may well be the most potent and specific inhibitor of FLT3 currently in development. A phase I study has recently been completed, studying activity in both FLT3 wild type(WT) and ITD relapsed and refractory AML.Seventy-six patients were treated on one of two 
schedules: intermittent (day 1-14) or continuous (day1-28) dosing. Pharmacokinetic studies revealed a prolonged plasma half-life of $\sim 36 \mathrm{hrs}$ and excellent ex-vivo target inhibition at dose levels above $12 \mathrm{mg}$ per day. Additionally an active metabolite was found, which likely contributes significantly to the biologic activity of AC220. The dose limiting toxicity was QTc prolongation at $300 \mathrm{mg}$ continuous dosing. The Phase II study of quizartinib was preliminarily reported, evaluating $90 \mathrm{mg}$ per day in females and $135 \mathrm{mg}$ per day in males in a continuous dosing strategy. ${ }^{10}$

\section{Ponatinib}

Recent pharmacological research has revealed ponatinib that is a multiple tyrosine kinase inhibitor and potent pan-BCR-ABL inhibitor. In preclinical models, ponatinib also inhibits the FLT3/ITD mutant prevalent in AML with potency similar to that of BCR-ABL. ${ }^{11} A$ cohort of patients with relapsed refractory AML were enrolled in a phase I trial of ponatinib ( $45 \mathrm{mg}$ once daily) to assess safety and preliminary efficacy in this population. Shah et al. published the results. ${ }^{12}$ Twelve patients were enrolled with a median age of 49 years. All patients had a history of a FLT3 mutation. Mutational analysis in a central laboratory confirmed the presence of FLT3-ITD in seven patients. The ORR was $3 / 12$ (25\%). Two patients achieved a complete remission with incomplete blood count recovery and one patient experienced partial remission. These three responders carried FLT3-ITD mutations and were all FLT3 inhibitor-naïve.

\section{Selumetinib (AZD6244)}

Selumetinib is an orally available MEK kinase inhibitor. The results of a multi-center phase II study of selumetinib in advanced AML. ${ }^{13}$ The dose used in this study was $100 \mathrm{mg}$ twice daily. Fortyseven patients were enrolled and the median number of cycles received was one. Grade $\geq 3$ adverse drug reactions included fatigue, dyspnea and nausea. One patient had partial response, three patients had minor response and two patients had unconfirmed minor response. In addition, four patients had stable disease. However, no patient with FLT3 ITD or NRAS mutation responded. This data indicates that it does have anti-leukemic activity but activity against FLT3 is not proven. ${ }^{14}$

\section{Crenolanib (CP-868,596)}

Crenolanib is a potent and selective type I FLT3 inhibitor. Smith and co-workers hypothesized that crenolanib may be a Type I inhibitor of FLT3 that retains activity against FLT3 mutant isoforms, including AC220-resistant FLT3 AL mutants, which are highly cross-resistant to multiple FLT3 TKIs. They published their results and confirmed that crenolanib is a Type I inhibitor as it bound preferentially to the phosphorylated form of $\mathrm{ABL}$. It also demonstrated substantially more potent in vitro binding affinity for the compound FLT3-ITD/D835V mutant than AC220. Crenolanib induced apoptosis and inhibited the proliferation of the patient-derived FLT3-ITD+ cell lines. ${ }^{15}$ 


\section{Post-transplant and other maintenance strategies}

Allogeneic transplant carried out in first remission appears to be the most effective conventional strategy for curing FLT3-ITD AML Even after CR with induction therapy, there is high likelihood to relapse, a short duration of remission, with relapse before a donor can be found. Therefore challenges with these patients include maintaining remission long enough to undergo transplant, and suppression of the growth of any leukemia clone still present after a transplant.

Several case reports have documented somewhat durable remissions to sorafenib when given to patients relapsing post-transplant. ${ }^{16}$

Clinical trials are ongoing investigating the utility and safety of quizartinib, sorafenib and midostaurin post allogeneic transplant. ${ }^{17}$

\section{Factors affecting clinical FLT3 targeting efficacy and associated resistance mechanisms}

Preclinical studies evaluating small molecule inhibitors of FLT3 were described shortly after the discovery of FLT3 mutations in AML. Through these studies it was revealed that the timing of incorporation of FLT3 inhibitors into therapy is predicted to influence clinical efficacy due to the cell cycle arresting characteristics of FLT3 inhibition. The ligand of the FLT3 receptor, FL, is found at peak levels 15 days after chemotherapy.

This ligand surge during aplasia renders the FLT3 ITD receptors more resistant to all FLT3 inhibitors and the surge appears to increase in levels with each subsequent cycle of cytotoxic chemotherapy. The surge of FL with each successive cycle as a potential resistance factor is an important consideration regarding the early incorporation of transplantation in FLT3-ITD leukemia. ${ }^{18}$ It is unclear if ITD length is associated with sensitivity to FLT inhibitor therapy but there appear to be biologic changes that occur with long ITD insertions. ${ }^{19}$

Resistance to agents targeting FLT3 ITD has been well described and can develop rapidly. The most common change associated with resistance is the acquisition of a point mutation in the kinase domain (D835Y)(19) or others. Other mechanisms have been described including up-regulation of anti-apoptotic pathways such as MCL-1or stromal response signaling cascades such as CXCR4.A retrospective study of patients receiving off label sorafenib upon relapse, however, revealed that only few developed resistance to sorafenib when given in the post-transplant setting $(47 \mathrm{v} 38 \% \mathrm{p}=0.03) .{ }^{17}$ 


\section{References}

1. Jemal A, Siegel R, Xu J, et al. Cancer statistics. CA Cancer J Clin. 2010; 60:277-300.

2. Becker H, Marcucci G, Maharry K, et al. Mutations of the Wilms tumor 1 gene (WT1) in older patients with primary cytogenetically normal acute myeloid leukemia: a Cancer and Leukemia Group B study. Blood. 2010; 116:788-92.

3. Takahashi S. Current findings for recurring mutations in acute myeloid leukemia. J Hematol Oncol. 2011; 4:36.

4. Weisberg E, Barrett R, Liu Q, et al. FLT3 inhibition and mechanisms of drug resistance in mutant FLT3positive AML. Drug Resist Updat. 2009; 12:81-9.

5. Zheng R, Bailey E, Nguyen B, et al. Further activation of FLT3 mutants by FLT3 ligand. Oncogene. 2011; 30:4004-14.

6. Stone RM, DeAngelo DJ, Klimek V, Galinsky I, Estey E, Nimer SD, et al. Patients with acute myeloid leukemia and an activating mutation in FLT3 respond to a small-molecule FLT3 tyrosine kinase inhibitor, PKC412. Blood. 2005;105(1):54-60.

7. Levis M, Brown P, Smith BD, Stine A, Pham R, Stone R, et al. Plasma inhibitory activity (PIA): a pharmacodynamic assay reveals insights into the basis for cytotoxic response to FLT3 inhibitors. Blood. 2006;108(10):3477-83.

8. Zhang W, Konopleva M, Shi YX, McQueen T, Harris D, Ling X, et al. Mutant FLT3: a direct target of sorafenib in acute myelogenous leukemia. J Natl Cancer Inst. 2008;100(3):184-98.

9. Pratz KW, Cho E, Levis MJ, Karp JE, Gore SD, McDevitt M, et al. A pharmacodynamic study of sorafenib in patients with relapsed and refractory acute leukemias. Leukemia. 2010;24(8):1437-44.

10. Levis MJ, Perl AE, Dombret H, Dohner H, Steffen B, Rousselot P, et al. Final Results of a Phase 2 Open-Label, Monotherapy Efficacy and Safety Study of Quizartinib (AC220) in Patients with FLT3-ITD Positive or Negative Relapsed/Refractory Acute Myeloid Leukemia After Second-Line Chemotherapy or Hematopoietic Stem Cell Transplantation. ASH Annual Meeting Abstracts. 2012;120(21):673.

11. Gozgit JM, Wong MJ, Wardwell S, et al. Potent activity of ponatinib (AP24534) in models of FLT3-driven acute myeloid leukemia and other hematologic malignancies. Mol Cancer Ther. 2011; 10:1028-35.

12. Shah NP, Talpaz M, Deininger MW, et al. Ponatinib in patients with refractory acute myeloid leukaemia: findings from a phase 1 study. Br J Haematol. 2013; 162:548-52.

13. Jain N, Curran E, lyengar NM, et al. Phase II study of the oral MEK inhibitor selumetinib (AZD6244) in advanced acute myeloid leukemia (AML). J Clin Oncol. 2012; 30.

14. Akinleye A, Furqan M, Mukhi N, et al. MEK and the inhibitors: from bench to bedside. J Hematol Oncol 2013;6:27. 
15. Smith CC, Lasater E, Mccreery M, et al. Crenolanib (CP-868,596) Is a Potent and Selective Type I FLT3 Inhibitor That Retains Activity Against AC220 Resistance-Causing FLT3 Kinase Domain Mutants. ASH Annual Meeting and Exposition 2012;141.

16. Sharma M, Ravandi F, Bayraktar UD, Chiattone A, Bashir Q, Giralt S, et al. Treatment of FLT3-ITD-positive acute myeloid leukemia relapsing after allogeneic stem cell transplantation with sorafenib. Biol Blood Marrow Transplant. 2011;17(12):1874-7.

17. Metzelder SK, Schroeder T, Finck A, Scholl S, Fey M, Gotze K, et al. High activity of sorafenib in FLT3-ITDpositive acute myeloid leukemia synergizes with alloimmune effects to induce sustained responses. Leukemia. 2012;26(11):2353-9. Reports of durable remissions with sorafenib for patients with FLT3-ITD AML in the post allogeniec transplant setting.

18. Kelly LM, Yu JC, Boulton CL, Apatira M, Li J, Sullivan CM, et al. CT53518, a novel selective FLT3 antagonist for the treatment of acute myelogenous leukemia (AML) Cancer Cell. 2002;1(5):421-32.

19. DeAngelo DJ, Stone RM, Heaney ML, Nimer SD, Paquette RL, Klisovic RB, et al. Phase 1 clinical results with tandutinib (MLN518), a novel FLT3 antagonist, in patients with acute myelogenous leukemia or high-risk myelodysplastic syndrome: safety, pharmacokinetics, and pharmacodynamics. Blood. 2006;108(12):367481.

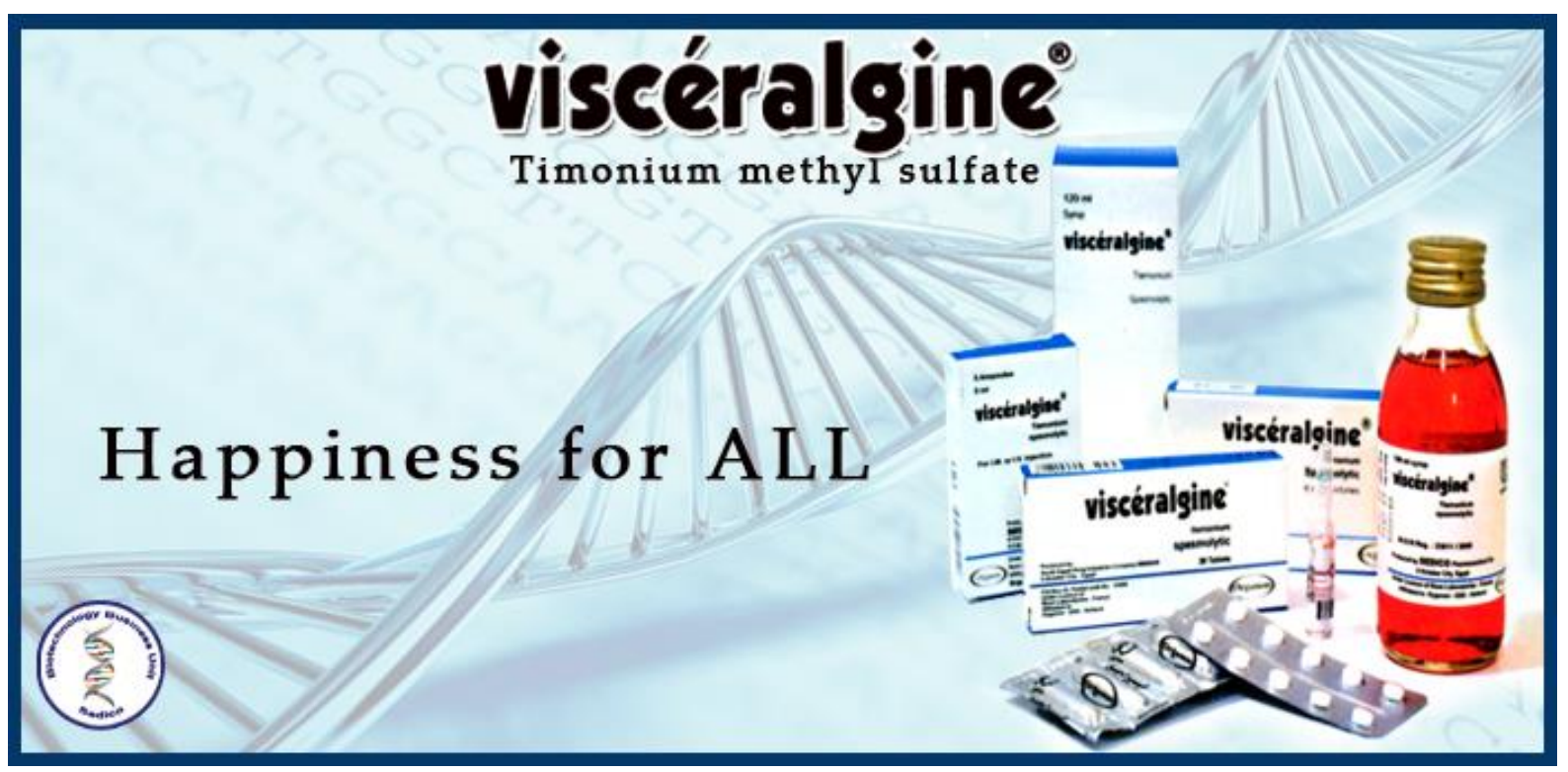

